\title{
К ТЕОРИИ СИНТЕЗА МНОГОСЛОИНЫХ ДИЭЛЕКТРИЧЕСКИХ СВЕТОФИЛЬТРОВ
}

Обобщен и дополнен предложенный в $\left.{ }^{1}\right]$ метод синтеза многослойных диэлектрических светофильтров.

\section{Введение}

В статье [ ${ }^{1}$ ] был развит новый метод синтеза многослойных диэлектрических светофильтров. Его существо заключается в нахождении с помощью преобразования Фурье такого непрерывного распределения показателя преломления в слое, при котором слой имеет заданную спектральную кривую пропускания. Затем непрерывный показатель преломления аппроксимируется системой дискретных слоев с такими толщинами и показателями преломления, чтобы ступенчатая кривая возможно точно имитировала непрерывную кривую. Там же было отмечено, что метод допускает в одной и той же задаче синтеза большое количество различных вариантов решения. Эту свободу можно использовать в целях подбора наиболее целесообразных (с точки зрения технологии) значений искомых показателей преломления слоев. В настоящей статье рассмотрим тот же метод в более общем виде и дополним его некоторыми новыми подходами, частично реализующими указанную свободу.

\section{Спектральная характеристика}

В основе нашего метода лежат выведенные в $\left[{ }^{2}\right]$ формулы для амплитудных коэффициентов отражения $r$ и пропускания $t$ неоднородного слоя. Эти формулы имеют вид бесконечных рядов

$$
\begin{aligned}
& \frac{r}{t} \exp \left(\frac{i k x}{2}\right)=a_{1}+a_{3}+a_{5}+\ldots, \\
& \frac{1}{t} \exp \left(-\frac{i k x}{2}\right)=1+a_{2}+a_{4}+\ldots,
\end{aligned}
$$

где $k$ - волновое число, $x$ - удвоенный оптический путь света:

$$
x=2 \int_{0}^{z} n(u) d u
$$

взятый от нижней границы $z=0$ слоя; $z$ - геометрическая координата, 
$n$ - показатель преломления. Свет падает в отрицательном направлении оси $z$ на верхнюю границу слоя, которая может лежать при любом значении $z$. Члены разложений выражаются в виде интегралов

$$
\begin{aligned}
a_{2 l+1} & =\int_{0}^{x} d a\left(x_{1}\right) \int_{0}^{x_{1}} d a^{*}\left(x_{2}\right) \ldots \int_{0}^{x_{2 l}} d a\left(x_{2 l+1}\right), \\
a_{2 l} & =\int_{0}^{x} d a^{*}\left(x_{1}\right) \int_{0}^{x_{1}} d a\left(x_{2}\right) \ldots \int_{0}^{x_{2 t-1}} d a\left(x_{2 l}\right),
\end{aligned}
$$

где

$$
a \equiv a_{1}=\int_{0}^{x} v(x) \exp (i k x) d x
$$

H

$$
v(x)=\frac{d n}{d x} \frac{1}{2 n} .
$$

Интеграл в формуле (5) имеет вид интеграла Фурье. Обратив его, мы выразим функцию $v(x)$ через $a(k)$. Но $a(k)$ можно приближенно связать с заданной спектральной кривой пропускания $T(k)$. Так, в $\left[{ }^{2}\right]$ было принято

$$
a a^{*} \approx \frac{1}{2}\left(T^{-1}-T\right)
$$

Однако это не единственно возможный и, вероятно, не лучший выбор. Покажем, что при том же порядке точности в правой части равенства (7) может стоять иная функция от $T$, причем возможных вариантов неограниченно много.

Прежде всего выведем соотношения, связывающие члены разложений (1). Они вытекают из энергетического соотношения

$$
\frac{1}{t t^{*}}-\frac{r r^{*}}{t t^{*}}=1
$$

где $t t^{* *}=T$ и $r r^{* *}=R-$ энергетические коэффициенты пропускания и отражения. Подставляя сюда разложения (1) и приравнивая члены с одинаковой кратностью интегралов, находим

$$
\sum_{m=0}^{l} a_{2 m} a_{2 l-2 m}^{*}=\sum_{m=1}^{l} a_{2 m-1} a_{2 l-2 m+1}^{*}
$$

где $a_{0}=1$ и $l=1,2, \ldots$ Те же соотношения вытекают из того, что при $x=0$ они, в силу формул (3)-(5), тривиально выполняются, а производные обеих частей равенств (9) тождественно равны; последнее следует из формул

$$
\begin{aligned}
& \frac{d a_{2 l+1}}{d x}=a_{2 l} \frac{d a}{d x}, \\
& \frac{d a_{2 l}}{d x}=a_{2 l-1} \frac{d a^{*}}{d x} .
\end{aligned}
$$

Используя теперь соотношения (9), выразим коэффициент пропускания в виде ряда

$$
T=1-a a^{*}-\left(A_{4}-\left(a a^{*}\right)^{2}\right)+\ldots,
$$

где временно обозначено

$$
A_{4}=a a_{3}^{*}+a^{*} a_{3} \text {. }
$$


Далее, пусть $S[k, T(k)]$ есть некоторая, пока произвольная функция, зависящая от $k$ отчасти прямо и отчасти через заданную функцию $T(k)$. Разлагая $S$ в ряд Тейлора вблизи значения $T=1$, с учетом (11) находим

$$
\begin{gathered}
S(k, T)=S(k, 1)-a a^{*}\left(\frac{\partial S}{\partial T}\right)_{T=1}- \\
-\left[\left(A_{4}-\left(a a^{*}\right)^{2}\right)\left(\frac{d S}{d T}\right)_{T=1}-\frac{\left(a a^{*}\right)^{2}}{2}\left(\frac{\partial^{2} S}{\partial T^{2}}\right)_{T=1}\right]-\ldots
\end{gathered}
$$

Потребуем далее, чтобы

$$
\begin{aligned}
& S(k, 1)=0, \\
& \left(\frac{\partial S}{\partial t}\right)_{T=1}=-1 .
\end{aligned}
$$

Тогда

$$
S(k, T)=a a^{*}+\left[A_{4}-\left(a a^{*}\right)^{2}+\frac{\left(a a^{*}\right)^{2}}{2}\left(\frac{\partial^{2} S}{\partial T^{2}}\right)_{T=1}\right]+\ldots
$$

Если ограничимся здесь первым членом разложения (т. е. членом второго порядка), то

$$
a a^{*} \approx S[k, T(k)]
$$

Таким образом, в пренебрежении членами четвертого и высших порядков любую функцию $S[k, T(k)]$, удовлетворяющую условиям (14), можно положить равной $a a^{*}$. Точность этого приближения определяется в основном членом четвертого порядка, зависящим, согласно (15), от второй производной $\left(\frac{\partial^{2} S}{\partial T^{2}}\right)_{T=1}$. Целесообразно выбирать $S[k, T(k)]$ так, чтобы этот член был в среднем по спектру возможно мал. Однако наперед об этом судить трудно, так как $a_{3}$ неизвестно. Исходный выбор остается поэтому произвольным, но указанное требование может быть учтено при итерации. Заметим, что введение в $S$ аргумента $k$ наряду с $T(k)$ тоже имеет целью облегчить наилучший выбор.

Итак, первым шагом к синтезу светофильтра, обладающего заданной кривой пропускания $T(k)$, является выбор спектральной характеристики $S[k, T(k)]$, удовлетворяющей условиям (14) и связанной с величиной $а$ приближенным равенством (16).

\section{Применение преобразования Фурье}

Обозначив

$$
\sqrt{S[k, T(k)]}=Q(k),
$$

согласно формулам (5), (6) и (16) получим

$$
\int_{0}^{x} \frac{d n}{d x} \frac{1}{2 n} \exp (i k x) d x=Q(k) \exp [i \varphi(k)]
$$

где $\varphi(k)$ - фаза, выбор которой, вообще говоря, произволен, но должен гарантировать вещественность $n(x)$. Равенство (18) является приближенным, если считать $n(x)$ заданным. Но мы задаем, наоборот, правую часть и рассматриваем это равенство как точное, определяющее некоторую функцию $n(x)$ такую, что принятие ее в качестве показателя 
преломления слоя реализует заданный коэффициент пропускания в силу того же равенства приближенно.

Перепишем формулу (18) с бесконечными пределами интегрирова-

ния (поскольку вне слоя $\left.\frac{d n}{d x}=0\right)$ :

$$
\int_{-\infty}^{+\infty} \frac{d n}{d x} \frac{1}{2 n} \exp (i k x) d x=Q(k) \exp [i \varphi(k)]
$$

Это - интеграл Фурье, обращая который, находим

$$
v(x)=\frac{d n}{d x} \frac{1}{2 n}=\frac{1}{2 \pi} \int_{-\infty}^{+\infty} Q(k) \exp [i(\varphi(k)-k x)] d k .
$$

Полученная формула дает принципиальное решение задачи синтеза. Конкретное применение ее было показано в $\left.{ }^{1}\right]$. Здесь мы обсудим эту формулу с общей точки зрения и установим некоторые новые возможности ее использования.

1. Зависимость $v(x)$ от фазы $\varphi(k)$. Если представим $v(x)$ в виде суммы четной $v_{p}(x)$ и нечетной $v_{n}(x)$ функций

$$
v(x)=v_{p}(x)+v_{n}(x),
$$

то, учитывая также условие вещественности $v(x)$, из формулы получим:

$$
\begin{aligned}
& v_{p}(x)=\frac{1}{2 \pi} \int_{-\infty}^{+\infty} Q(k) \cos \varphi(k) \cos k x d k, \\
& v_{n}(x)=\frac{1}{2 \pi} \int_{-\infty}^{+\infty} Q(k) \sin \varphi(k) \sin k x d x,
\end{aligned}
$$

причем должны тождественно выполняться равенства

$$
\begin{aligned}
& \int_{-\infty}^{+\infty} Q(k) \sin \varphi(k) \cos k x d k=0, \\
& \int_{-\infty}^{+\infty} Q(k) \cos \varphi(k) \sin k x d k=0 .
\end{aligned}
$$

Из этих равенств следует, что $Q(k)$ должно быть четной функцией, а $\varphi(k)$ или $\varphi(k) \pm \pi-$ нечетной. Если подвергнем фазу преобразованию $\varphi(k) \rightarrow-\varphi(k)$, то, как видно из формул $(22), v_{p}(x)$ не изменится, а $v_{n}(x)$ переменит знак. Если же сделаем $\varphi(k) \rightarrow \pi-\varphi(k)$, то $v_{p}(x)$ переменит знак, а $v_{n}(x)$ не изменится. Наконец. если положим $\varphi(k) \rightarrow \pi+\varphi(k)$, то и $v_{p}(x)$, и $v_{n}(x)$ переменят знак. Таким образом, в зависимости от выбора фазы мы получаем различные решения. Конечно, возможны, кроме указанных преобразований, и другие способы варьирования фазы. Частным случаем является выбор постоянной фазы, т. е. $|\varphi(k)|=\varphi=$ const, причем условие нечетности $\varphi(k)$ требует, чтобы $\varphi(-k)=-\varphi(k)=-\varphi$. Тогда формулы (22) принимают вид: 


$$
\begin{aligned}
& v_{p}(x)=\frac{\cos \varphi}{\pi} \int_{0}^{\infty} Q(k) \cos k x d k, \\
& v_{n}(x)=\frac{\sin \varphi}{\pi} \int_{0}^{\infty} Q(k) \sin k x d k .
\end{aligned}
$$

2. Синтезируя светофильтр, мы обычно интересуемся поведением пропускания не во всем спектре, а только в ограниченном его участке. Обозначим его через $\left[k_{1}, k_{2}\right]$. Это обстоятельство сильно расширяет возможности выбора функции $Q(k)$, поскольку вне интересующего нас участка вид $T(k)$ остается совершенно произвольным; следовательно, одной и той же функции $S[k, T(k)]$ соответствует множество функций $Q(k)$. Целесообразно эту свободу использовать все-таки иным способом. Вычислив по формуле (20) функцию $v(x)$ при какой-либо заданной функции $Q(k)$, мы можем заменить первую функцией

$$
v_{1}(x)=v(x)+\eta(x),
$$

где

$$
\eta(x)=\sum_{j} c_{j}\left\{\delta\left(x-x_{j}\right)-\frac{\sin \left[k_{2}\left(x-x_{j}\right)\right]-\sin \left[k_{1}\left(x-x_{j}\right)\right]}{\pi\left(x-x_{j}\right)}\right\},
$$

$c_{j}$ - произвольные числа и $x_{j}$ - произвольно фиксированные (возрастающие с индексом) значения координаты $x$. Как легко убедиться, функция $\eta(x)$ обладает свойством

$$
\int_{-\infty}^{+\infty} \eta(x) \exp (i k x) d x=0, \quad \text { если } k<k_{1} \quad \text { или } k>k_{2} .
$$

Это значит, что $v_{1}(x)$ дает в интервале $\left[k_{1}, k_{2}\right]$ то же значение $a$, что и $v(x)$, и следовательно, ту же кривую пропускания $T(k)$ (с той же степенью точности). А так как согласно формуле (6)

$$
n(x)=\exp \left(2 \int_{-\infty}^{x} v(u) d u\right)
$$

то замена $v(x)$ на $v_{1}(x)$ приводит к замене $n(x)$ на

$$
n_{1}(x)=N(x) n(x),
$$

где

$$
N(x)=\exp \left(2 \int_{-\infty}^{x} \eta(u) d u\right)
$$

Подставляя сюда вместо $\eta(u)$ выражение $(26)$, находим

$N(x)=\exp \left(2 \sum_{j=1}^{m} c_{j}\right) \prod_{j} \exp \left\{\frac{2 c_{j}}{\pi}\left[\operatorname{Si}\left[k_{1}\left(x-x_{j}\right)\right]-\operatorname{Si}\left[k_{2}\left(x-x_{j}\right)\right]\right]\right\}$,

где $m$ определяется условием $x_{m}<x<x_{m+1}$. Первый множитель в этой формуле отражает дискретное изменение $N(x)$ в точках $x_{j}$ (что обусловлено присутствием $\delta$-функции в формуле (26). Таким образом, если показатель преломления реализует в интервале частот $\left[k_{1}, k_{2}\right]$ заданную кривую $T(k)$, то такую же кривую реализует приближенно и показатель преломления $n_{1}(x)$. В этом случае слой состонт из нескольких частей, внутри которых показатель преломления изменяется 
непрерывно, а на границах испытывает скачки, величина которых зависит от чисел $c_{j}$. Изложенный прием может быть полезен для корректировки показателя преломления в тех случаях, если нужно его уменьшить или увеличить в том или другом промежутке.

\section{Заключение}

Итак, мы показали, что предложенный в [ $\left.{ }^{1}\right]$ метод синтеза диэлектрических светофильтров обладает большой общностью и допускает варьирование решения в широких пределах. Изложенным не исчерпываются возможности метода. Дальнейшее развитие его и, в частности, вопросы, связанные с итерированием, будут рассмотрены в последующих статьях.

\section{ЛИТЕРАТУ Р А}

1. С ос си Л., Изв. АН ЭССР, Физ. Матем., 23, 229 (1974).

2. С осс и Л., К ард П., Изв. АН ЭССР, Физ. Матем., 17, 41 (1968).

\section{Тартуский государственный} университет
Поступила в редакцию $24 / \mathrm{VI} 1975$

\section{SOSSI}

\section{MITMEKIHILISTE DIELEKTRILISTE VALGUSFILTRITE TEOORIAST}

Artiklis [ $\left.{ }^{1}\right]$ esitatud interferentsfiltrite sünteesi meetodi üldistusena näidatakse, et Fourier' teisendusvalemis (18) on võimalik funktsiooni $Q(k)$, kus $k$ on lainearv, valida lõpmata mitmeti. On ainult nõutav, et sellega seotud funktsioon $S[k, T(k)]=Q^{2}(k)$ (valem (17)), kus $T(k)$ on etteantud läbilaskvustegur, rahuldaks tingimusi (14). Valiku kitsendamine täpsema sünteesi huvides on võimalik ainult iteratsiooni kaudu. Juhul kui sünteesitaval filtril peab etteantud spektraalkõver $T(k)$ olema piiratud spektrivahemikus $k_{1}, k_{2}$, on vôimalik korrutada murdumisnäitajat $n(x)$ teguriga $N(x)$ (valem (31), kus $c_{j}$ ja $x_{j}$ on meelevaldsed), ilma et $T(k)$ selles vahemikus oluliselt muutuks.

\section{SOSSI}

\section{ON THE THEORY OF THE SYNTHESIS OF MULTILAYER DIELECTRIC LIGHT-FILTERS}

As a generalization of the method of the synthesis of interference light-filters described in $\left.{ }^{1}\right]$, it is shown that the function $Q(k)$ in the Fourier transformation formula (18), where $k$ is the wave number, is to a large extent arbitrary. It is only required that the involved function $S[k, T(k)]=Q^{2}(k)$ (formula (17)), in which $T(k)$ is the transmittance given in advance, should satisfy the conditions presented by (14). A limitation of this arbitrariness and, accordingly, a more accurate synthesis is solely possible by iteration. If the filter must have the given transmittance $T(k)$ only in the restricted spectral interval $\left[k_{1}, k_{2}\right]$, then the refractive index $n(x)$ can be transformed by a factor $N(x)$ (formula $(31)$, where $c_{j}$ and $x_{j}$ are arbitrary), without any essential change of $T(k)$ in this interval, 